\title{
SOBRE LA PRONUNCIACIÓN DEL ESPAÑOL EN EL ECUADOR
}

Desde que Gustavo Lemos publicó, hace treinta años, sus Barbarismos fonéticos del Ecuador, pocos hechos nuevos se han aducido a propósito de la pronunciación ecuatoriana. Existen minuciosos estudios formales (fonéticos sobre todo) sobre el español de varias zonas de Hispanoamérica: Nuevo México, México, Yucatán, Puerto Rico, Santo Domingo, Colombia, Chile, la Argentina, el Paraguay, el Perú, y aun sobre la lengua criolla de Curaçao (el papiamento) ${ }^{1}$, pero el Ecuador se cuenta entre aquellas regiones -Cuba, Centroamérica, Panamá, Venezuela, Uruguay, Bolivia y las provincias amazónicas de Colombia y el Perú- cuyas características lingüísticas no se han descrito aún de manera completa ${ }^{2}$. Los apuntes que aquí ofrecemos vienen a resumir ( $\mathrm{y}$ a interpretar en ciertos casos) los datos sueltos que hasta la fecha tenemos sobre la geografía fonética ecuatoriana ${ }^{3}$.

1 Merecen citarse sobre todo los siguientes: Nuevo México: A. M. EspinosA, El español de Nuevo Méjico, BDH, vols. I y II; México: El español en Méjico, los Estados Unidos y la América Central, BDH, vol. IV (trabajos de E. C. Hills, F. Semeleder, C. C. Marden, M. G. Revilla, A. R. Nykl, K. Lentzner, C. Gagini y R. J. Cuervo, con notas y estudios de P. HenríquezUreña), J. Matluck, La pronunciación del español en el Valle de México, y P. Boyd-Bowman, El españól de Guanajuato (en prensa); Yucatán: Víctor M. Suárez, El español que se habla en Yucatán; Puerto Rico: T. Navarro, El español en Puerto Rico; Santo Domingo: P. Henríquez-Ureña, El español en Santo Domingo, BDH, vol. V; Colombia: R. J. Cuervo, Apuntaciones criti. cas sobre el lenguaje bogotano, y L. FLórez, La pronunciación del español en Bogotá; Chile: $E l$ español en Chile, $B D H$, vol. VI (trabajos de R. LeNz, A. Bello y R. Oroz); Argentina: E. Tiscornia, El lenguaje del "Martín Fierro", $B D H$, vol. III, y B. E. VIDAL DE BATTINI, El habla rural de San Luis, BDH, vol. VII; Paraguay: B. Malmberg, Notas sobre la fonética del español en el Paraguay; Perú: P. Benvenutto Murrieta, El lenguaje peruano; papiamento: R. LENz, El papiamento, [y T. NAVARro, "Observaciones sobre el papiamento", en este mismo volumen de la $N R F H]$.

${ }^{2}$ No deja de ser curioso, en el campo de la dialectología hispánica, el hecho de que en la Península misma los dialectos periféricos (gallego, asturiano, leonés, extremeño, andaluz, murciano, aragonés, etc.) han sido objeto de frecuentes investigaciones lingüisticas, mientras que casi nada se ha hecho hasta ahora sobre el habla rural de las dos Castillas.

${ }^{3}$ Al regresar de México a Boston en el verano de $195^{2}$ tuve por compañeros de viaje a tres ecuatorianos que venían a conocer los Estados Unidos: un joven 


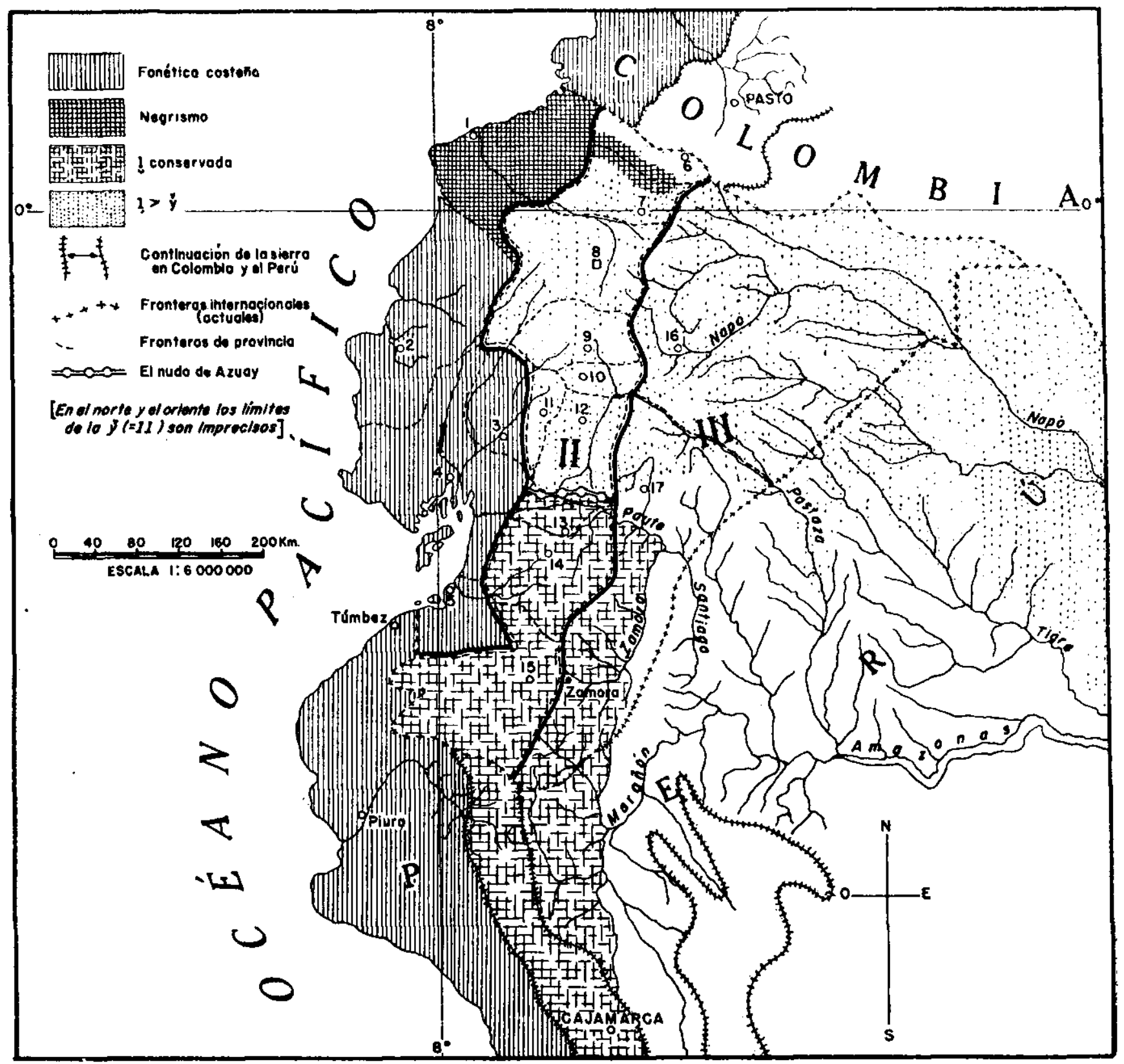

MAPA Geográlico-i,ingǘístico del Fcuador.-I. La Costa: I. Esmeraldas (capital de la provincia de Esmeraldas); 2. Portoviejo (id. de Manabi); 3. Babahoyo (de Los Ríos); 4. Guayaquil (de Guayas); 5. Machala (El Oro). II. La Sierra: 6. Tulcán (Carchi); 7. Ibarra (Imbabura); 8. Quito (Pichincha); 9. Latacunga (León); 10. Ambato (Tungurahua); 11. Guaranda (Bolívar); 12. Riobamba (Chimborazo); 13. Azogues (Caìar); 14. Cuenca (Azuay); 15. Loja (Loja). III. El Oriente: 16. Tena (Napo-Pastaza); 17. Macas (Santiago-Zamora).

Según los datos a nuestro alcance, el Ecuador puede dividirse en tres zonas dialectales: I) la costa (con Guayaquil), que desde el punto de vista fonético forma parte de la vasta y antigua zona marítima de influencia negra y andalucista (las Antillas; la costa mexicana de Veracruz, Tabasco y Campeche; Panamá y partes de Centroamé-

hacendado (Napoleón Solórzano, 24 años, de Quito), una empleada del gobierno ecuatoriano (Mariana Dousdebés, 22 años, quiteña también) y una maestra de escuela (Victoria Valenzuela, 30 años, de Guayaquil). Ninguno de ellos sabía inglés. Durante tres días fuí apuntando a cada paso mis observaciones sobre su pronunciación e interrogándoles a menudo sobre las peculia. ridades lingüisticas de las regiones del Ecuador que ellos conocían directamente. En cuanto a los ejemplos referentes a Cuenca, Loja y Cañar, debo mucho también a don Julio C. Vinueza, de Cuenca, quien con suma gentileza llenó el cuestionario que le envié acerca del habla de su región. 
rica; las costas de Venezuela, Colombia, Ecuador, Perú y centro de Chile); 2) la sierra (con Quito), que se vincula lingüísticamente con el Sur de Colombia y el Norte del Perú; 3) las provincias amazónicas. Dentro de la primera zona hay que señalar por su negrismo la provincia norteña de Esmeraldas, la cual penetra lingüísticamente en la sierra por una hondonada en que también predominan los negros, la Hoya del Chota. Dentro de la zona serrana se destacan en el Sur las provincias de Cañar, Loja y Azuay (con su capital, Cuenca), lleístas como la sierra del Perú4. Cañar y Cuenca ("la Atenas del Ecuador") se distinguen además por su singular canturreo esdrujulista (cúchillo, cástigo, Guáyaquil). Sobre la zona orien. tal (provincias de Napo-Pastaza en el Norte y Santiago-Zamora en el Sur) no tenemos informes. Región selvática de difícil acceso, ha sido colonizada desde la sierra a lo largo de la cuenca de los ríos Napo, Pastaza, Paute y Zamora ${ }^{5}$.

4 Emplearemos los términos lleismo (lleista), žeismo (žeista) para denotar, igual que yeismo (yeista), las distintas maneras de pronunciar la $l l$.

5 Según J. Morales, Ecuador. Nociones históricas. Geografía fisica y antrópica, Guayaquil, 1938, pág. 75, las provincias costeñas tenían, en 1997, un total de 1.256,00o habitantes, distribuídos como sigue: 386,000 blancos $(31 \%)$, 130 ,00o indios (10\%), 275,000 mestizos $(22 \%), 395,000$ mulatos $(31 \%)$ y 70 ,ooo negros $(6 \%)$. (Todos los porcentajes son míos). La sierra, con 2.172, ooo habitantes, comprendía 598,000 blancos $(26 \%), 692,000$ indios $(32 \%)$, 842,000 mestizos $(40 \%)$, y (principalmente en el Valle del Chota) 32,000 mulatos (1.6\%) y 8,0oo negros (o.4\%). El Oriente, con sólo 188,000 habitantes, tenía 10,000 blancos $(5.3 \%), 163,000$ indios $(86.7 \%), 15,000$ mestizos $(8 \%)$, sin negros ni mulatos. Sumando las tres zonas, resulta que, sobre una población total de $3.616,000$ habitantes, en 1937 había 994 ,ooo blancos $(27.5 \%), 985,000$ indios $(27.2 \%), 1.132,000$ mestizos $(31.3 \%), 427,000 \mathrm{mu}-$ latos $(11.8 \%)$ y 78 ,ooo negros $(2.2 \%)$. Hay que observar que desde 1937 pasaron a poder del Perú el puerto y el distrito costeño de Túmbez, el territorio de Jaén (políticamente parte de la provincia serrana de Loja) y unas tres cuartas partes de todo el Oriente; pero las cifras arriba citadas nos dan de todos modos una buena idea de las proporciones étnicas del país. Los negros y mulatos, poco numerosos, están concentrados en la provincia costeña de Esmeraldas (población, 68,0oo habitantes) y en el valle serrano del Chota. Los indios de la sierra, humildes y sumisos a los blancos, abundan en las provincias septentrionales (Imbabura, Pichincha, León, Tungurahua, Chimborazo), especialmente en las dos primeras, donde viven en caseríos cerca de las ciudades o diseminados en los campos. Al Sur, en Loja y Azuay, los indios, menos numerosos, "viven mezclados con el blanco". "Los indios del Azuay, donde el mestizaje es intenso, hablan generalmente el castellano y son bastante civilizados por su contacto con los blancos y mestizos" (Morales, op. cit., págs. 74 y 100). También los indios de la costa, los llamados montuvios, han venido a mezclarse con los blancos, sobre todo en la gran provincia de Guayas (530,00o habitantes) . A diferencia de los humildes indios serranos, los montuvios son "arrogantes y soberbios, pretensiosos y pendencieros" (Morales, pág. 74). En el Oriente había en 1937, sobre un. total de 163,000 indios, unos 20,00o quechuas, la mayoría de ellos en el Norte, cerca de la sierra. Los jívaros, numerosos en otro tiempo, se hallan confinados hoy a las riberas de los ríos Santiago, Morona y Pastaza en el Sur. Hablan su propio idioma. En la sierra, según Morales (pág. 
Examinemos, ante todo, algunos rasgos fonéticos de las dos primeras zonas:

La 11.-La costa es yeísta; la sierra distingue entre $l l$ y $y^{6}$. Las provincias meridionales de Loja, Azuay y Cañar forman una unidad con la zona lleísta del Perú (lave, val̦e; yo, mayo), pero el resto de la sierra (con Quito, la capital) opone a la y no la l̦, sino una ž que, según Amado Alonso, sería mejor representar por $\breve{y}$, puesto que la articulación correspondiente a la $l l$ es "rehilada, casi siempre fricativa, sin avanzamiento hacia el predorso y los alvéolos" " . Como advertí en mi nota "Sobre restos de lleísmo en México" (NRFH, VI, 1952, págs. 69-74), la $l l$ ecuatoriana tiene que haber llegado a $\check{z}$ (o y) sin pasar por la etapa confundidora y. Por lo tanto, esta y no puede tener el mismo origen que la $\check{z}$ argentina $(<l l, y)$. Los quiteños distinguen sin dificultad entre poy̆o 'pollo' y poyo 'poyo', entre olla y hoya, calló y cayó, etc. Sería interesante fijar la frontera septentrional entre la $\breve{y}$ ecuatoriana y la 1 colombiana, ya que los departamentos hoy colombianos de Nariño y Cauca, también distinguidores en su parte serrana (FLórEZ, pág. 240), dependían en la época colonial de la Real Audiencia de Quito. Además, convendría estudiar los dialectos quechuas en el Ecuador para determinar si en las regiones en que la $l l$ castellana ha pasado a $\breve{y}$, el cambio $1>$ y se ha realizado también en quechua.

Un hecho interesante es que la actual frontera entre la 1 y la $\breve{y}$ del Ecuador la forma el Nudo de Azuay, gran macizo que separa en dos porciones casi iguales la sierra ecuatoriana. Esta frontera coincide con otra política de la época incaica, la del reino de los cañaris, "enemigo de los quitos", que en la entrada de 1533-34 enseñaron el camino de Quito a los conquistadores españoles.

76), las "parcialidades indigenas conservan aún su melodioso quichua", mientras que en las selvas costeñas de Esmeraldas subsiste, como "dialecto muy reducido", el de los indios cayapas.

- Tanto en la sierra como en la costa se enseña en las escuelas una diferencia fonética entre la $l l$ y la $y$, pero en la costa ni siquiera los maestros logran distinguirlas correctamente en la práctica. La Srta. Valenzuela pronunciaba indiferentemente $\mathbf{y}$ o $\check{z}$, de preferencia esto último. El mismo rehilamiento del sonido $y$ se nota en el litoral del centro y Sur del Perú, sobre todo entre los jóvenes de Lima, donde Murrieta (pág. 122) lo atribuye al "contagio argentino trasmitido en el cine parlante y las tan difundidas grabaciones fonográficas de tangos y canciones". Pero yo creo que en el caso de Lima y Guayaquil, que son dos focos lingüísticos, podría tratarse más bien de una evolución hispánica independiente de la ž porteña y paralela a ella. Recordemos los múltiples focos de difusión que tuvieron el seseo y el yeísmo primitivos.

"Amado Alonso, "La $l l$ y sus alteraciones en España y América", en EMP, II, pág. 67 .

' Cf. J. Rumazo, La región amazónica del Ecuador en el siglo xvi, Sevilla, 1946, pág. 3. 
Es muy posible que esta antigua frontera política y natural constituya también una frontera dialectal dentro del quechua; si resulta que el paso $1>\breve{y}$ se ha realizado en quechua al Norte del Nudo de Azuay pero no al Sur, entonces ya no cabrá duda de que la y̌ del español de la sierra quiteña se relaciona con la fonética quechua en el habla de aquella región bilingüe. Puede ser que la provincia oriental de Napo-Pastaza, colonizada desde la sierra norteña, comparta también su y distinguidora. Murrieta, pág. 128 (citando al geógrafo y misionero fray Francisco Cheesman Salinas, Los jivaros, Lima, 1930), nos proporciona un dato precioso: no sólo los indígenas del monte, sino también la plebe mestiza de los pueblos y de ciudades como Iquitos (ocupada en esa época por el Perú y cedida más tarde a este país) sustituyen la $l l$ por $c h$ : achá 'allá'. (De la y no dice nada, lo cual nos da a entender que el cambio afecta solamente a la $l l$ ). Creo que la grafía $c h$ es una representación aproximada, de acuerdo con los recursos de la ortografía española, del mismo sonido y̆ que Jorge IcAzA, en Huasipungo, identifica con $s h$. A juzgar por otros cambios que Cheesman Salinas refiere a esta región amazónica: $d>t$ o $t t-n a t a$, natta 'nada', ticho, tticho 'dicho', tte 'de' - , $b, v>p$ - pien 'bien', lloper (sic por choper) 'llover' - , no sorprendería una emisión explosiva, o por lo menos sorda, de la $l l$ también: alá $>$ al̂yá $>$ alcká (con 1 ensordecida) $>$ aĉá. Según me escribe don Julio C. Vinueza, en la provincia oriental de Zamora hay mezcla de l y $\breve{y}$, con predominio de la primera.

Como lo demuestra Amado Alonso en su magistral artículo "La $l l$ y sus alteraciones...", el moderno yeísmo hispánico arranca de la segunda mitad del siglo xvir. Parece que se desarrolló espontáneamente en España y América, observándose primero en las grandes ciudades (Lima lo tenía antes que Sevilla). Pero no tenemos derecho para deducir que el yeísmo sea esencialmente un fenómeno urbano y la conservación de la $l l$ un arcaísmo rural. Hay regiones yeístas nada urbanas (por ejemplo Nuevo México), y en cambio hay ciudades de primera importancia (Asunción, Cusco, Quito, Bogotá) que conservan la $l l$.

Consideremos ahora la posible influencia (o sea apcyo) de las lenguas indígenas. En América el fonema 1 sobrevive, como vemos, casi exclusivamente en regiones bilingües de influencia quechua, mapuche y guaraní. Pues bien, a diferencia del náhuatl mexicano (lengua que siempre ha carecido de este fonema), el quechua y el mapuche tenían su $l l$ en la época de la conquista, mientras que el guaraní la ha adoptado, primero en los hispanismos que entraron en su vocabulario, y luego en su propio sistema fonemático, por influencia del españot y posiblemente del quechua también. Aunque faltan pruebas concluyentes, es difícil no pensar en una correlación entre la $l l$ de estos idiomas y la conservación de la $l l$ en el es- 
pañol de las zonas bilingües ${ }^{9}$. Fuera de las zonas bilingües citadas, la $l l$ se conserva, por razones todavía no aclaradas, en Bogotá y parte de la sierra colombiana.

En resumen, hay una zona distinguidora entre la y y la $l l$ (pronunciada 1 o y), faja estrecha, pero continua, que corre a lo largo de los Andes desde el departamento de Norte de Santander (Colombia) hasta el Paraguay y Chile, donde la continuidad se ve interrumpida por el yeísmo de las grandes ciudades del centro.

Volviendo al Ecuador, la provincia negra de Esmeraldas (en la costa) tiende a perder la $y(<1, \mathbf{y})$ en contacto con $i$. Se oye maravia, gaina, poito, a $i$ 'alli', fenómeno que también se observa en la costa colombiana del Pacífico (Flórez, pág. 244) y en el Norte del Perú (Murrieta, págs. 122 y 129 , registra cuchio, gaina, caudio y aun botea 'botella').

La s.-La sierra ecuatoriana, como la del Perú, pronuncia una $s$ tenue y firme en cualquier posición. Era predorsal la de mis informantes quiteños. En el habla del joven Solórzano noté que la $s$ a veces ensordecía y asimilaba una vocal precedente tras oclusiva sorda: est's, cuant's, ant's, crio k'si. Tales pronunciaciones se dan a menudo en el habla rápida de la altiplanicie mexicana, cuya $s$ predorsal, como la $s$ andina, se mantiene fuerte y aguda siempre ${ }^{10}$.

En cambio, la costa aspira la $s$ final de sílaba y de palabra y la pierde en final de grupo fónico: lah cohta, doh fóhforo, ìno supihteh eso? (En nohotro la -s- también se aspira, sin duda por persistir la conciencia del origen compuesto de este pronombre). La maestra guayaquileña pronunciaba, a diferencia de los quiteños, una $s$ coronal dental plana de poca tensión muscular.

La $\mathrm{r}$ (tr, rr).-Es notable la tendencia serrana hacia la fricación y ensordecimiento parcial de la ápicoalveolar vibrante ${ }^{11}$. Formada entre el ápice y los alvéolos y bastante parecida a la $s$ ápicoalveolar castellana, la $r$ fricatica conserva entre vocales o ante consonante sonora su sonoridad, pero ante sorda o en final de grupo fónico hay varios grados de ensordecimiento ${ }^{12}$. Apunto como ejemplo con $r r$ :

9 La $l l$ paraguaya, aprendida y exagerada por los indígenas hasta el punto de ser hoy más enérgica aún que la $l l$ peninsular, me recuerda un caso algo parecido, el de la $-s$ final latina, que se esforzaban por pronunciar, como quienes aprendían un idioma extranjero, los hispanos y los galos, mientras los italianos y los rumanos, hablando el latín como lengua patrimonial, la iban relajando.

10 Véase mi nota "La pérdida de vocales átonas en la altiplanicie mexicana", en NRFH, VI, 1952, págs. $13^{8-140 .}$

11 Como en el departamento limítrofe de Nariño (Colombia). Cif. Flórez, pág. 233.

12 En América, la $r r$ asibilada se oye en Nuevo México, México, Guatemala, Colombia, Ecuador, el Perú, Bolivia, Paraguay, Uruguay, Chile y las provin- 
tierra, carro, rojo, reloj, iqué horror!; con tr o dr: tres, cuatro, otro, tren, trigo, tiatro (cf. Bacha, forma hipocorística de Beatriz), padre, madre, Pedro; con -r: mejor, calor, horror, popular, fértil, norte, parte, puerco, perla, carne, Hernando. A veces la fricación alcanza hasta la $-r$ - intervocálica: caro, puro, Tire 'Tere ( $<$ Teresa)'.

Para la costa, mis datos son menos precisos. Entre los montuvios o campesinos de la costa, y sobre todo en la provincia negra de Esmeraldas, tanto la $-r$ como la $-l$ finales de palabra aguda tienden a desaparecer: peó, muhé 'mujer', señó, trabajá, animá 'animal', pa$p \dot{e}$; mientras que en sílaba átona ante consonante se confunden, unas veces en $l$ (polque si, pol mi mare), las más veces en $r$ (arma 'alma', arguno, ivárgame Dió!, er polvo, er camino $)^{13}$. Pero en Guayaquil, según insistía la Srta. Valenzuela, la $l$ y las vibrantes $r$ y $\overline{\mathrm{r}}$ (nunca fricativas) se distinguen bien, por lo menos entre personas que tengan un poco de educación. Hasta se refuerza la $-r$ en posición final (¿como reacción contra su pérdida?) con vibraciones múltiples: calo $\overline{\mathbf{r}}$, vivi $\overline{\mathbf{r}}$. En la profesora guayaquileña observé tras $-r$ final ante pausa una especie de eco de la vocal acentuada precedente: vivire, muhere, pagar $^{a}$, flor ${ }^{o}$, sur ${ }^{o}$. Eco parecido (color $^{e}$, ehcribir $\left.{ }^{i}\right)$ lo he oído a un señor veracruzano en México ${ }^{14}$. Se podría interpretar en ambos casos como una vocal de apoyo agregada a la $-r$ final absoluta en regiones donde ésta tiende a caer.

cias andinas de la Argentina. En Costa Rica se asibila el grupo tr: chonco 'tronco' (C. GaGini, Diccionario de costarriqueñismos, pág. 8). En España la asibilación ocurre en Álava, Rioja, Navarra y Aragón (cf. FlókEz, pág. 233; Vidal de Battinı, "Extensión de la $r r$ múltiple en la Argentina", Fil, III, 1951, págs. 181-184, y LeNz, en $B D H$, VI, págs. 99 y 115 ).

${ }_{13}$ Así en Andalucía y Extremadura, en las Antillas, Panamá, Curaçao (papiamento) y las costas de Venezuela, Colombia y el Perú (mujé, singulá, generá, arza, sarsa, carculá, seportura: Murrikta, págs. 129, 112, 121). En el centro de Chile, $-r$ da $-l$ en posición final absoluta o en final de palabra ante vocal, $y$ "en interior de palabra $l$ y $r$ dan distintos resultados según la naturaleza de la consonante siguiente" (A. Alonso y R. LidA, "Geografía fonética: -l y $-r$ implosivas en el español”, RFH, VII, 1945, pág. 322 ; cf. Flórez, págs. 200 y sigs.). Un alumno de mi curso de lingüística hispanoamericana, Lloyd K. Hulse, me comunica que en la costa de Veracruz la - $r$ final sólo se pierde en los infinitivos ante $l$ (decile). En la conversación rápida y descuidada $-l$ y $-r$ pueden borrarse y confundirse fonéticamente, pero en la mente del hablante no dejan de distinguirsc, y cn una pronunciación más lenta y menos relajada vuelven a adoptar su forma propia. Tal vez haya que rectificar, pues, en algunos casos, la categórica afirmación de Alonso y Lida (loc. cit., pág. 324) de que la alternancia de $r$ y $l$ indica "un único fonema intermedio u oscilante -único e idéntico en su valor intencional de signo, e idéntico también en la mente de los hablantes".

14 Lloyd K. Hulse (cuyo estudio sobre la pronunciación de Veracruz aparecerá en uno de los próximos números de $N R F H$ ) confirma esta observación y me dice que tras $-r$ final absoluta se oye en Veracruz, en personas de cualquier edad, un elemento vocálico indefinido, beber ${ }^{e}$, vivir ${ }^{i}$ (pero no ${ }^{*}$ color $^{\circ}$ ), que entre los ancianos de otros pueblos de la costa suena siempre e: tomare, calor $^{e}$, ayere . 
La n velar.-Tanto la sierra como la costa pronuncian como velar la - $n$ final de palabra ante pausa, ante vocal, ante nasal, ante $l$, y por supuesto ante velar: Juay, estación, nos day otro, en $u_{1}$ avión militar, uyas (por influencia de uy), cọ nohotro, ey Méhico, coymigo, uy limón, e y lugar de, uy jovey, uy gray coyquistador. Esta y, articulación relajada de la consonante nasal acompañada de mayor o menor nasalización de la vocal precedente, es un fenómeno muy difundido en todo el mundo hispánico. En la Península, la -n velar aparece en los dialectos del Oeste y del Sur (gallego, asturiano, leonés, extremeño, andaluz), y en América la encontramos en las Antillas, en la costa mexicana de Tamaulipas, Veracruz, Tabasco y Campeche, en los estados de Puebla, Morelos, Guerrero, Oaxaca y Chiapas, en Guatemala y Nicaragua, en las zonas marítimas de Venezuela, Colombia y el Perú, en la costa y la sierra del Ecuador y en todo el Río de la Plata. Es de suponer que en Panamá, Costa Rica, Salvador y Honduras también se dé el fenómeno, aunque falta confirmación ${ }^{14}$ bis. Las regiones americanas que no velarizan. la $-n$ son Nuevo México y México (salvo la costa y el Sur), Chile (donde por lo menos la -1 no se daba en tiempos del gran fonetista Lenz) y las sierras de Colombia, el Perú y tal vez Bolivia.

Algunos grupos cultos.-En su habla normal, todos mis informantes convertian en velar la oclusiva labial de los grupos consonánticos: sectiembre, octener, adacta, eseksión 'excepción', ocservar, y la maestra guayaquileña también las alveolares y dentales: igmenso, igsmo ${ }^{15}$, egsétera, agmóhfera, arigmética, con $\stackrel{0}{\mathbf{g}}$ ensordecida implosiva. Los serranos pronunciaban admósfera, aridmética, izmo, como en el español normal. El quiteño Napoleón Solórzano no pronunciaba el grupo $t l$. Decía at-las, $A$-clántico. Con las otras dos personas no hice pruebas.

La š.-Conocida es la facilidad con que la gente inculta de México pronuncia la š de palabras nahuas, aunque la š (o $\check{\mathbf{z}}$ ) de los anglicismos la suele convertir en ch (chainiar 'shine', Orench Crach 'Orange Crush'), y de la š española antigua no hay ni el más pequeño recuerdo. De igual manera los serranos del Ecuador y del Perú pronuncian sin dificultad la š, pero. casi exclusivamente en indigenismos quechuas ${ }^{16}$.

14 bis Últimamente nos llega confirmación de la - $n$ velar en El Salvador: $p a_{1]}$, andén, e $e_{\mathfrak{y}}$ aktomóvil (D. L. CANFIELd, HispB, XXXVI, 1953, págs. 32-33).

15 En esta palabra se trata, evidentemente, de una transposición de consonantes: istmo $>$ idsmo, forma esta última que recogí en Guanajuato (México), junto con logaridsmo y aridsmética, y que se ha registrado además en Guatemala (Sandoval, Semántica guatemalteca, I, pág. 736) y en Colombia (Currvo, Apuntaciones, $\$ \$ 810$ y 837 ).

${ }_{10}$ En cuanto a la š en el Perú, véase Murrieta, pág. 127. 
$L a$ j y la f.-En la costa, pero no en la sierra, la $j$ suena muy débil, convirtiéndose en una mera aspiración faríngea: diho 'dijo', muhere (o muhé), Huạ, Méhico, hovẹ. Este debilitamiento consonántico caracteriza una vez más las regiones marítimas de influencia andaluza o negra (Andalucía, las Antillas, la costa de Veracruz, etc.). Los quiteños pronuncian la $j$ velar, pero más suave que la castellana, más o menos como en la altiplanicie mexicana. Lo mismo que en el habla rural de casi toda Hispanoamérica, sobrevive aquí la aspiración procedente de la $f$ - primitiva en algunas palabras, pero identificada con la pronunciación local de la $j$, por ejemplo juir, jodido 'lastimado', jarto, jeder, joya, 'hoya', jondo, jacer, jablar, jembra, jumo, jilo, jigado (entre los indios jigádo), jaba, jorno, jervir, jipar, josco. No se oye hierro, sino como en México, jierro o fierro. En el habla vulgar y rural puede sonar $\mathbf{x}$ también la $f$-moderna, sobre todo ante semiconsonante: jué, juerza, juerte, juego 'fuego', juera, juente, jiesta, jiebre, jierrocarril (sic), Rajel, jebrero, conjesarse, ajeitarse, injinito, dijicil, jósjoro (entre indios josjóro, entre el vulgo de Cuenca también jósporo, que recuerda el fósporo colombiano de FLórez, pág. 171), jlores, Jrancisco, ojrecer. Como caso de ultracorrección tengo fuez 'juez' (Cuenca).

Ante las vocales abiertas a y e (pero no ante o), la $f$ se oye a veces, al menos entre el pueblo de Cuenca, como aspiración velar emitida con aproximación de los labios: juácil, juamilia, Rajuel. En realidad no se trata aquí de una sucesión de dos sonidos, sino de un sonido compuesto, sentido como único por el hablante, por lo cual sería quizá preferible representarlo en lo futuro por un solo signo, digamos 'w (w aspirada).

El fenómeno se ha observado también en Tabasco, México (BDH, IV, pág. 339) y en la costa colombiana del Pacífico, donde FLórez (pág. 182) lo atribuye sólo a negros y mulatos.

La aspiración de la $f$ se ha registrado en Nuevo México, México, Guatemala, Honduras, Costa Rica, Santo Domingo, Puerto Rico, Venezuela, el Ecuador, la Argentina, Canarias, Oriente de Asturias, Occidente de Salamanca, Sur de España, Extremadura leonesa, Maragatería y Astorga, Santander y en aragonés (FLórEZ, pág. 180). La $f$ de mis informantes, personas cultas los tres, vacilaba entre bilabial y labiodental (Felipe, fácil, flor, fiesta, fuman, profundo, fósforo, aunque ante $\mathbf{w}$ parecía ser más seguramente bilabial (fué, fuerza, fuego). La $f$ bilabial se ha registrado en Nuevo México, Guatemala, Costa Rica, Puerto Rico, el Perú, Chile, el Paraguay, Extremadura, en localidades de la Sierra de Gata y de Andalucía, esporádicamente en Navarra (FLórEZ, pág. 171$)^{17}$.

17 La interpretación de la primitiva $f$ castellana como bilabial (o bilabial fuertemente aspirada) es la que tal vez mejor explica fonéticamente el paso $\mathbf{f}>\mathbf{h}>$. En apoyo de esta interpretación está el hecho de que el castellano ha 
La b, la d y la g.-En los ambientes rurales de la sierra persiste la fórmula de tratamiento vusted, como en gran parte de la sierra colombiana (por lo menos en los departamentos de Nariño, Tolima, Cundinamarca, los Santanderes y Boyacá) (Flórez, pág. 141). Es general en la costa la pérdida de la - $d$ final de palabra: verdá, uhté, etc. La - $d$ - intervocálica suele caer en la pronunciación vulgar o semiculta de la costa, sobre todo entre los negros: naa, too, toavia, dao, parao, peazo 'pedazo', mario, cansáa, comio, coy er grao e capitáł. Los negros también pronuncian a veces mare, pare, pero no sé si se extiende o no a otras palabras con $d r$, como cuadro, Pedro, vidrio ${ }^{18}$. En cambio, la sierra ecuatoriana, cuyo consonantismo

rechazado siempre, pese al esfuerzo cultista, la pronunciación labiodental de la $v$. El que la $v$ sonora se haya mantenido como bilabial mientras la $f$ sorda se ha mostrado tan inestable se explicaría por la adecuada perceptibilidad de la primera. En cambio, es notorio que la perceptibilidad de una fricativa bila. bial sorda es casi nula. Fisiológicamente, hay dos modos de vencer esta debilidad acústica:-retirando ligeramente el labio inferior para hacer silbar la corriente de aire por entre los dientes superiores (con lo cual se cambia el timbre), o apartando un poco los labios para dar al sonido bilabial una articulación más enérgica (es decir, con más fuerza expiratoria), sin abandonar, con un notable cambio de timbre, su oposición fonemática a la $v$ bilabial sonora.

Si, como creemos, el castellano primitivo adoptó realmente la segunda solución, entonces suponemos una lenta transformación del fonema $f$ con las siguientes etapas: I) una bilabial con fricación ligera pero fuertemente aspirada, cuyo valor de signo residiría al principio en su sordez frente a la $\mathbf{v}$, en su carácter bilabial frente a las otras fricativas sordas (s, ̌̌s), sirviendo la fuerza expiratoria sólo para prestar al sonido un grado mayor de perceptibilidad; 2) una fuerte aspiración faríngea ligeramente bilabializada (este cambio seria más bien psicológico que fonético: el valor de signo se habría trasladado ahora a la misma aspiración faríngea, quedando la bilabialidad, ahora rasgo secundario, como simple recuerdo de la antigua oposición homorgánica $\mathbf{f}: \mathbf{v}$ ); y 3) la aspiración faríngea, por sí sola, olvidado ya el origen bilabial.

La $f$ apoyada a) en líquida: flor, frio, frente $<*$ fruente (con $f$ bilabial, a juzgar por la pérdida por disimilación de la bilabial w), b) en la semiconsonante $\mathbf{w}$ : fuerza, fuente (con fusión del grupo $f \mathbf{w}$ en un solo sonido bilabial aspirado y redondeado ' $w$ ), y $c$ ) (con menos regularidad) en la semiconsonante $\mathbf{j}$ (fiero, fiesta, contra hierro, hiel), se habría mantenido como bilabial a través de aquella época en que la aspiración procedente de $\mathbf{f}$ ante vocal iba perdiéndose en el habla popular castellana. Pero más tarde se habría reforzado hasta la $f$ apoyada, optando el castellano por el refuerzo labiodental (cediendo tal vez a la pronunciación de otros dialectos), mientras que el andaluz y el extremeño, como el habla vulgar y rústica de parte de América, la habría convertido en una aspiración faríngea $\dot{h}$. Con el tiempo, esta $\dot{h}$ hubiera sufrido la misma suerte que la $\mathbf{h}$ (ya perdida) procedente de la $\mathbf{f}$ en otras posiciones, de no haberse confundido muy pronto con la recién aparecida aspiración velar $\mathbf{x}$ procedente de las antiguas ̌̌ y ̌̌. En las regiones de consonantismo relajado, esta confusión se resolvió en $\dot{h}$, en otras en $\mathbf{x}$.

18 Entre hablantes negros del litoral de Colombia se ha observado mare (Flórez, pág. 151); pare en Nuevo México y Colorado (Espinosa, BDH, I, pág. 167); pae, mae (y pai, mai) en las Antillas y la costa colombiana del Ca. 
(como el de la sierra del Perú y el del Valle de México) se mantiene firme, conserva no sólo la - $d$ - fricativa intervocálica -hablado, soldado, colorada, nada, todo, toditico (hasta la gente vulgar pronuncia así)-, sino también la - $d$ final entre la gente culta y semiculta: sed, salud, ciudad, verdad. La fórmula de tratamiento usted se oye al lado del vulgar usté (o vusté) y del rústico su mercé. En el Perú la gente culta de la sierra, pero no la de la costa, pronuncia la - $d$ final (Murrieta, pág. 110). Lo mismo ocurre en el Valle de México.

Vocalismo.-Por influencia del quechua, los indios y chagras (mesțizos) de la sierra suelen confundir, al hablar castellano, las vocales $e-i, o-u$. En el Huasipungo de Jorge ICAzA pueden verse muchos ejemplos, aunque siempre en boca de indios ${ }^{19}$ : istu 'esto', shurandu 'llorando', di dundi 'de donde', tinimus 'tenemos', socres 'sucres', etc. La misma indiferencia vocálica no sólo caracteriza en el Perú la fonética serrana vulgar, sino que afecta a menudo las capas superiores "por natural contagio". Se oyen en la sierra peruana formas como mojir 'mujer', obriro, tentiro 'tintero', amego 'amigo', mantiquilla (Murrieta, pág. 123). Hay que advertir que en mis dos informantes quiteños no se acusaba la menor confusión ${ }^{20}$.

ribe: mare, pare, mae, pae en Andalucía (L. Rodrígufz-Castellano y A. PaLAcIo, "El habla de Cabra", RDTP, IV, 1948, pág. 578); el fenómeno ocurre, pues, en regiones de consonantismo relajado. En portugués, como se sabe, la forma normal es mãe. En francés, lengua que en su evolución fonética a partir del latín ha mostrado tan marcada tendencia a relajar y perder sus consonantes intervocálicas y finales, la pérdida del elemento dental del grupo -TR- o -DR- se ha realizado siempre: pierre, verre, lierre, carrefour.

${ }_{19}$ Con la intención puramente artística de señalar con rasgos fonéticos cl habla pintoresca de los indios, a ellos principalmente refiere Icaza tanto el vocalismo cerrado como el sonido que él representa con $s h(<l l)$ : posho, vashe, ashi, gashinita. En cuanto al lenguaje de los chagras, se concreta a indicarlo con algunos detalles morfológicos y léxicos (voseo; frecuente empleo de indigenismos; partículas ca, miso; el arcaísmo yo vide; el sufijo-tico). Pero a veces se descuida: hace hablar con la $s h$ a un chagra (shevar, Guashabamba 'Guaillabamba'), o, cansado de seguir indicando la fonética india, representa el diálogo de los indios con la ortografía del español normal (cebolla). Así, pues, las indicaciones de Icaza sobre la extensión social de los fenómenos del habla de la sierra deben recibirse con mucha cautela.

${ }^{20}$ En Nariño y Cauca (departamentos colombianos contiguos al Ecuador) se han registrado cincu, quietu, digu, jondu, cabestru, cascorvu, Inaciu, demuñu, tíu, primu, riu, buenu, enfermu, peru, muchachu, ¿cómu sigue? (FLórez, págs. 77-78). Pero quizá no se trate aquí de influencia quechua, en primer lugar porque el fenómeno "es frecuente y muy perceptible" también en Antioquia, Caldas y Norte de Santander, y en segundo lugar porque en Nariño y Cauca no se dan casos del cambio $-e>-i$ (sí hay algunos en Caldas, más al Norte). Dice Flórez (pág. 77): "el oscurecimiento [de o átona en $u$ ] se advierte hasta en el lenguaje cuidado de personas cultas, y no sólo en final de palabra ante pausa, sino en vocablo interior de frase". Y prosigue: "A antioqueños y caldenses muy cultos, y a algunos bogotanos también, he oído varias veces en 
En cuanto a las vocales en hiato, la gente vulgar de la sierra tiende a romper el hiato: Elóisa, tiatro, pior, rial, Napolión, Lionardo, aura (aurita) 'ahora (ahorita)', maistros cai, trair, trairás, pais, maiz, raiz. Ejemplos de Loja: pior, piones; de Cuenca (gente inculta): óimos, cáido, léido, Raféil y Rajuel (pron. Ra'wel, con bilabiovelar aspirada), maiz, pais, raiz. Ejemplos de ultracorrección (sólo entre indios, según don Julio C. Vinueza) son caé, caye, creyen, leyer. (Todas estas formas, con la posible excepción de Raféil y Rajuel, se dan también en ambientes rurales de la altiplanicie mexicana). Las formas en ai se oyen a menudo con $e i$, y viceversa, sobre todo entre indios: Raféil, Rajuel, Rajel (a través de Rafail), reiz, treir, queir (y aun trer, quer), como en la sierra del Perú (Murrieta, pág. 115), paine, sais. En cambio, la costa mantiene por lo general el hiato: traemo, peor o peó (entre negros), „Dió se lo páe! ‘Dios se lo pague!', maí, rai. La pronunciación costeña demuestra igual firmeza vocálica oponiendo su pueh (o pué) al pes o ps serrano.

Acentuación.-Como en la sierra peruana (Murrieta, pág. 123), los indios de la sierra ecuatoriana suelen hacer llanas tanto las palabras esdrújulas como las agudas: sabádo, miercóles, ultimo, jigádo, articúlo, pildóra, arbóles, periodico, pajáro; Órtiz, Escóbar, capúli, Guayáquil ${ }^{21}$. En cambio, cuando imploran a los patrones o cuando rezan, los indios siempre desplazan a la última sílaba el acento de las fórmulas de tratamiento: amitú 'amito', siñoritá, taiticú 'taitico, Diosito'.

En Cuenca y Cañar, aunque no en Loja, prevalece un curioso canturreo que desplaza el acento hacia la primera sílaba. Este modo de hablar lo llaman les cuencanos "esdrujulizar" o "hablar esdrújulamente": cástigo, cúchillo, ábrigo, Alberto, cántamos, véndemos, Guáyaquil, libertad, cordillera. Excepción hecha de los indios, el fenómeno es bastante general. En cuanto a su origen, tratándose de una antigua y rica ciudad universitaria, "la Atenas del Ecuador", no se puede descartar la posibilidad de un desarrollo exagerado, mantenido entre el pueblo por reacción contra la mencionada acentuación llana de los indios, de la manía esdrujulizante que a principios del siglo pasado sufrió la lengua general (intérvalo, méndigo). El asunto merece estudiarse más a fondo. Ejemplos con el acento en la primera pero no antepenúltima sílaba: úniversidad (no *univérsidad), périodico, Dómblas 'Don Blas'. Según me avisa el Sr. Vi-

Bogotá pronunciar como $u$ más o menos abierta la o final de palabra (y cn la terminación -os), en discursos, lecturas y conferencias públicas".

${ }_{21}$ Al parecer, este desplazamiento del acento no afecta a la desinencia verbal. Los indios no dicen, por ejemplo, *quedo por quedó, ni *diran por dirán. 
nueza, "la manía de esdrujulizar va desapareciendo en la gente culta y queda únicamente en el vulgo".

Conclusiones.-Los apuntes anteriores demuestran claramente la continuidad fonética entre las costas de Colombia, el Ecuador y el Perú (yeísmo, relajamiento de ${ }^{i} y-,-y^{i},-s,-r,-l,-d,-d-, j$, pero con vocalismo firme) frente a la de sus provincias andinas (conservación de la oposición $l l: y$, tal vez por influencia quechua, y de las consonantes intervocálicas y finales; $\overline{\mathbf{r}}, t r,-r$ fricativas o asibiladas, diptongación de los hiatos, y, en el caso del Ecuador y del Perú, influencia fonética del quechua por lo menos en la acentuación, el vocalismo y la pronunciación de la š).

Dentro de la sierra del Ecuador notamos que las tres provincias meridionales (Loja, Azuay, Cañar) pronuncian la $l l$ como 1 , mientras que al Norte del Nudo de Azuay (y tal vez en la provincia oriental de Napo-Pastaza) la $l l$ suena y̆. En el Norte, según queda dicho, hay un valle poblado de negros, la Hoya del Chota, que pertenece lingüísticamente a la provincia negra de Esmeraldas, en la costa.

Para el estudioso de la historia lingüística hispanoamericana, lo que más debe importar son las condiciones geográficas, étnicas, históricas y sociológicas. Desarrollada a lo largo de los tres siglos de la época colonial, la dialectalización de la América española tiene poco que ver con las actuales fronteras políticas, de creación posterior y a menudo arbitrarias.

Por ejemplo, las fronteras actuales del Ecuador con los países colindantes no corresponden ni a fronteras naturales (esto es, geográficas), ni a fronteras lingüístico-culturales (se habla el mismo español de ambos lados), ni a fronteras políticas antiguas (incaicas o coloniales). En el siglo xvir el territorio de la Real Audiencia de Quito abarcaba Pasto y Popayán, llegaba por el Norte hasta San Buenaventura y Cali, y hacia el Sur y el Este, además de llegar a Túmbez, comprendía toda la región amazónica que hoy es territorio peruano.

Es de esperar, pues, que el que profundice en el estudio crítico de la fonética ecuatoriana (española y quechua) lo combine con un estudio serio de las fonéticas del Norte del Perú y del Sur de Colombia, tratadas hasta ahora casi únicamente como si fuesen desviaciones del habla de Lima o de Bogotá. 